\title{
THE CIRCUMSTELLAR ENVIRONMENT OF $\sigma$ ORIONIS E
}

\author{
C. IAN SHORT AND C. T. BOLTON \\ David Dunlap Observatory, University of Toronto \\ Richmond Hill, Ontario, L4C \&YG, CANADA
}

\section{Introduction}

The light and spectrum of the magnetic, chemically peculiar Be star $\sigma$ Orionis E (=HD 37479) vary with the rotational period of $1.19081 \mathrm{~d}$ because of the effects of inhomogeneities in the photosphere and circumstellar matter (Walborn and Hesser 1976, Landstreet and Borra 1978, Hunger 1974). In this paper, we describe a model for the distribution of the $\mathrm{H} \alpha$ emitting material in the magnetosphere based on analysis of 24 high quality spectra covering the entire rotation period and published data from other sources.

\section{Geometrical and Astrophysical Constraints on the Gas Distribution}

There are two "eclipses" in the light curve that are caused by transits of dense clouds of circumstellar material across the stellar disk. These clouds are centered on the intersections of the magnetic and rotational equators at phase $\phi=0.0$ and $\phi=0.44$. The eclipse durations indicate that the clouds subtend $33^{\circ}$ and $47^{\circ}$ respectively at the star's center.

We have isolated the $\mathrm{H} \alpha$ line profiles of the circumstellar material by subtracting a photospheric profile derived by fitting the $\mathrm{H} \delta$ line profile with line blanketed model atmospheres. We obtain $T_{\text {eff }}=23250 \mathrm{~K}, \log g=4.0$, $N_{\mathrm{He}} / N_{\mathrm{H}}=1.0$, and $v \sin i=150 \mathrm{~km} \mathrm{~s}^{-1}$. If the clouds are rotating rigidly, the Doppler widths of the circumstellar profiles observed when the dense clouds are in quadrature indicate that the radial dimensions of the clouds are $R(\phi=0.0)=7.0 R_{\star}$ and $R(\phi=0.44)=6.4 R_{\star}$. The Doppler width of the emission is nearly constant for most of the rotational period, so there must be gas present at the maximum radial extent over a broad range of rotational longitude.

Circumstellar emission is observed at $\Delta \lambda= \pm \lambda_{\mathrm{H} \alpha} v \sin i / c$ when the clouds are at quadrature, so they are "attached" to the photosphere. The absorption cores near $\Delta \lambda=0$ observed during the eclipses indicate that the one of the clouds is occulted and one is transiting the stellar disk during each eclipse. Thus the clouds must be nearly co-planar and the inclination of the rotation axis must be $i \geq 82^{\circ}$. The emission seen near $\Delta \lambda=0$ for 
$0.55 \leq \phi \leq 0.88$ indicates that magnetic equator has a modest inclination, $\beta \simeq 20^{\circ}$, with respect to the rotational equator.

The shallow eclipses are either partial or else $\tau_{\text {cont }}<1$ in the clouds. If the eclipses are total and the continuous opacity is due to HI boundfree transitions and Thomson scattering, the wavelength dependence of the eclipse depths in the Paschen continuum, yield $N_{\mathrm{e}}(\phi=0.0) \geq 5.47 \pm 0.06 \times$ $10^{10} \mathrm{~cm}^{-3}$ and $N_{\mathrm{e}}(\phi=0.44) \geq 3.52 \pm 0.03 \times 10^{10} \mathrm{~cm}^{-3}$. Then the depth of the $u$-band eclipse yields $\tau_{\mathrm{H} \alpha}(\phi=0.0) \approx 3400$ in the radial direction. The blue (red) wings of the emission profiles obtained near $\phi=0.3 \mathrm{differ}$ from the red (blue) wings of profiles $0.5 P_{\text {rot }}$ later, so the clouds are also optically thick in the longitudinal coordinate. The clouds must be very thin perpendicular to the rotational equator because $N_{\mathrm{e}}$ derived from the last visible Balmer shell line during the eclipses (Groote and Hunger 1976) is 30 times greater than the lower limit derived from the light curve, and the $\mathrm{H} \alpha$ shell lines absorb $<10 \%$ of the photospheric flux. This suggests that $i=90^{\circ}$.

\section{A Preliminary Model}

These results show that the circumstellar gas is distributed in a thin disk with large blade-shaped clouds at the intersections of the magnetic and rotational equators. If $i=90^{\circ}$, the asymmetry in the line-of-sight magnetic field variation and the eclipse spacing can be explained by a symmetric magnetic dipole whose axis has a moderate inclination with respect to the rotational axis and center is displaced toward $\phi=0.8$ and downward toward the hemisphere containing the negative magnetic pole. The radiatively driven wind from a broad region around the magnetic equator is channeled into the magnetic equator by the magnetic field where it compresses the gas into a thin disk. Recombined hydrogen ions drift outward and backward in the disk relative to the co-rotating frame due to the effects of centrifugal acceleration, radiation pressure, and wind compression. After many ionizations and recombinations they enter the leading edge of one of the clouds at the potential minima (Nakajima 1981) centered on the intersections of the equators. We believe that this process can explain many of the details in the light curves as well as the dissimilarity of the leading edges and similarity of the trailing edges of the clouds.

\section{References}

Groote, D. and Hunger, K.: 1976, Astr. Ap. 52, 303

Hunger, K.: 1974, Astr. Ap. 32,449

Landstreet, J.D. and Borra, E.F.: 1978, Ap. J. Lett. 224, L5

Nakajima, R.: 1981, Tohoku Univ. Sci. Rept. 2, 129

Walborn, N.R. and Hesser, J.E.: 1976, Ap. J. Lett. 205, L87 